\title{
On Polynomial Solutions of Pell's Equation
}

\author{
Hasan Sankari ${ }^{1}$ and Ahmad Abdo $\mathbb{D}^{2}$ \\ ${ }^{1}$ Tishreen University, Faculty of Science, Department of Mathematics, Lattakia, Syria \\ ${ }^{2}$ Tartous University, Faculty of Science, Department of Mathematics, Tartous, Syria \\ Correspondence should be addressed to Ahmad Abdo; ahmad_abdo@tartous-univ.edu.sy
}

Received 7 June 2021; Accepted 6 August 2021; Published 12 August 2021

Academic Editor: Marco Fontana

Copyright (c) 2021 Hasan Sankari and Ahmad Abdo. This is an open access article distributed under the Creative Commons Attribution License, which permits unrestricted use, distribution, and reproduction in any medium, provided the original work is properly cited.

Polynomial Pell's equation is $x^{2}-D y^{2}= \pm 1$, where $D$ is a quadratic polynomial with integer coefficients and the solutions $X, Y$ must be quadratic polynomials with integer coefficients. Let $D=a_{2} x^{2}+a_{1} x+a_{0}$ be a polynomial in $\mathbb{Z}[x]$. In this paper, some quadratic polynomial solutions are given for the equation $x^{2}-D y^{2}= \pm 1$ which are significant from computational point of view.

\section{Introduction}

Let $D$ be a quadratic polynomial with integer coefficient. We consider polynomial Pell's equation

$$
x^{2}-D y^{2}= \pm 1
$$

where $X, Y$ are quadratic polynomials with integer coefficients. Many fruitful research studies have been conducted in this direction.

In 1976, Nathanson [1] proved that when $D=x^{2}+d$, equation (1) has a nontrivial solution if and only if $d= \pm 1, \pm 2$.

In 2001, Mollin et al. [2] described the background to the study of continued fraction expansions of $\sqrt{D}$ where $D$ is a polynomial, especially quadratic, which we study herein.

\section{Notation and Preliminaries}

Let $D$ be a given square-free natural number. It is well known that Pell's equation

$$
x^{2}-D y^{2}=1,
$$

always has an infinite number of integer solutions (see e.g. Nagell [3]). All the solutions of (2) with positive $x$ and $y$ are obtained by the formula $A_{n}+B_{n} \sqrt{D}=(a+b \sqrt{D})^{n}$ where $n=1,2,3, \ldots$, and $a+b \sqrt{D}$ is the fundamental solution of (2).

Pell's equation

$$
x^{2}-D y^{2}=-1
$$

may not always have integer solutions. When this equation is solvable in integers for some given $D$, if $L+M \sqrt{D}$ is its fundamental solution, we have the relation

$$
a+b \sqrt{D}=(L+M \sqrt{D})^{2},
$$

where $a+b \sqrt{D}$ is the fundamental solution of (2).

For a given value of $D$, in order to determine the fundamental solution of (2) or (3) (when it exists), one may use the method of continued fraction expansion of $\sqrt{D}$. The penultimate convergent of this expansion provides the fundamental solution.

From computational point of view, it would be advantageous to group the values of $D$ into several classes such that the values of $D$ in a specific class are associated with a similar pattern of continued fraction expansion. It is found that polynomial expressions for $D$ come in handy for this purpose.

As usual $\left[a_{0} ; \overline{a_{1}, \ldots, a_{n} ; 2 a_{0}}\right]$ will denote the simple infinite periodic continued fraction 


$$
a_{0}+\frac{1}{a_{1}+\left(1 / a_{n-1+\left(\cdot / a_{n+\left(1 /\left(2 a_{0}+\left(1 /\left(a_{1}+\cdot .\right)\right)\right)\right)}\right)}\right)} .
$$

$A_{i} / B_{i}$ will denote the $i^{\prime}$ th convergent:

$$
a_{0}+\frac{1}{a_{1}+\left(1 /\left(a_{2}+\cdots\left(1 / a_{i}\right)\right)\right)} .
$$

Use will also be made of the following:

$$
\begin{aligned}
& A_{i}=a_{i} A_{i-1}+A_{i-2}, \\
& B_{i}=a_{i} B_{i-1}+B_{i-2}, \\
& \frac{A_{-1}=B_{-2}=1, A_{-2}=B_{-1}=0, \text { where }}{A_{i} B_{i-1}-A_{i-1} B_{i}=(-1)^{i-1},}
\end{aligned}
$$

each of these being valid for $i=0,1,2, \ldots$.

Definition 1 (see [4]). A polynomial $f(t) \in \mathbb{Z}[t]$ is called a Fermat - Pell polynomial in one variable if there exists polynomial $c(t), h(t) \in \mathbb{Z}[t]$ such that

$$
c(t)^{2}-f(t) h(t)^{2}=1, \quad \text { for all } t \in \mathbb{Z} \text {. }
$$

A triple of polynomials $(c(t), h(t), f(t))$ satisfying equation (8) constitutes a polynomial solution to Pell's equation. A Fermat-Pell polynomial is said to have a polynomial continued fraction expansion if there exists an integer $T$ and a positive integer $n$ such that

$$
\sqrt{f(t)}=\left[a_{0}(t) ; \overline{a_{1}(t), \ldots, a_{n}(t)}\right]
$$

for all integral $t \geq T$, where $a_{i}(t) \in \mathbb{Z}[t]$. For a fixed Fermat - Pell polynomial $f(t)$, a pair of polynomials $(c(t), h(t))$ is said to be the fundamental polynomial solution to equation (9) if $(c(t), h(t))$ constitutes the smallest solution in positive integers to equation (8) for all non-negative values of $t \geq T$.

Definition 2 (see [5]). For real $x$, let the symbol $x$ denote the greatest integer $\geq x$.

Theorem 1 (see [5]). Suppose $D=4 t^{2}+12 t+5$, where $t$ is any natural number. Then, $a+b \sqrt{D}$ is the fundamental solutions of (2) where $a=4 t^{3}+18 t^{2}+24 t+9$, $b=2 t^{2}+6 t+4$.

Theorem 2 (see [5]). If $D=4 t^{2}+4 t+5$ where $t$ is any natural number, then $L+M \sqrt{D}$ is the fundamental solution of (3) where $L=4 t^{3}+6 t^{2}+6 t+2, M=2 t^{2}+2 t+1$.

Theorem 3 (see [6]). Let $D \geq 1$ be a non-square integer; then:

$$
\sqrt{D}=\left[a_{0} ; \overline{2 a_{0}}\right] \Leftrightarrow D=a_{0}^{2}+1,
$$

where $D$ is a positive integer.

\section{Main Results}

Let $D=a_{2} x^{2}+a_{1} x+a_{0}$ be a polynomial in $\mathbb{Z}[x]$. In this section, we will prove our main results. As a start, we record the following propositions.

Proposition1. Let $D=D(t)$, where $t$ is any natural number. If $\sqrt{D}=\left[a_{0}(t) ; \overline{2 a_{0}(t)}\right]$, then:

(1) $L+M \sqrt{D}$ is the fundamental solution for the equation $x^{2}-D y^{2}=-1$, where $L=a_{0}(t), M=1$.

(2) $a+b \sqrt{D}$ is the fundamental solution for the equation $x^{2}-D y^{2}=1$, where $a=2 a_{0}(t)^{2}+1, b=2 a_{0}(t)$.

Proof. Let us assume $\sqrt{D}=\left[a_{0}(t) ; \overline{2 a_{0}(t)}\right]$. Then:

(1) The penultimate convergent is

$$
\frac{a_{0}(t)}{1}
$$

Thus, we get the relation stated above, and by (10),

$$
L^{2}-D M^{2}=a_{0}(t)^{2}-\left(a_{0}(t)^{2}+1\right)(1)^{2}=-1 .
$$

(2) The penultimate convergent is

$$
a_{0}(t)+\frac{1}{2 a_{0}(t)}=\frac{2 a_{0}(t)^{2}+1}{2 a_{0}(t)} \text {. }
$$

Thus, we get the relation stated above, and by (10),

$$
a^{2}-D b^{2}=\left(2 a_{0}(t)^{2}+1\right)^{2}-\left(a_{0}^{2}+1\right)\left(2 a_{0}(t)\right)^{2}=1 .
$$

Theorem 4. Let $t, m, p$ be any natural numbers, $p$ be a prime, and $a+b \sqrt{D}$ be the fundamental solution of (2); then:

(1) If $D=m^{2} t^{2}+m$, then $a=2 m t^{2}+m, b=2 t$.

(2) If $D=p^{2} t^{2}+2 p$, then $a=p t^{2}+1, b=t$.

(3) If $D=m^{2} t^{2}+2 m t$, then $a=m t+1, b=1$.

(4) If $D=4 t^{2}+(4 m+4) t+m^{2}+m$, then $a=4 t+2 m+1, b=2$.

(5) If $D=16(m+1)^{2} t^{2}+8(m+1)^{2} t+m^{2}+m$, then $a=32(m+1) t^{2}+16(m+1) t+2 m+1, b=8 m+2$.

Proof. (1) We have

$$
m t<\sqrt{D}<m t+1
$$

and so $\sqrt{D}=m t$. Now, expanding $\sqrt{D}$ into a continued fraction expansion, we obtain 


$$
\begin{aligned}
\sqrt{D} & =m t+\sqrt{m^{2} t^{2}+m}-m t \\
& =m t+\frac{1}{\left(\left(\sqrt{m^{2} t^{2}+m}+m t\right) /\left(m^{2} t^{2}+m-m^{2} t^{2}\right)\right)} \\
& =m t+\frac{1}{2 t+\left(\left(\sqrt{m^{2} t^{2}+m}-m t\right) / m\right)} \\
& =m t+\frac{1}{2 t+\left(1 /\left(m\left(\sqrt{m^{2} t^{2}+m}+m t\right) /\left(m^{2} t^{2}+m-m^{2} t^{2}\right)\right)\right)} \\
& =m t+\frac{1}{2 t+\left(1 /\left(2 m t+\sqrt{m^{2} t^{2}+m}-m t\right)\right)} \\
& =[m t ; \overline{2 t, 2 m t]} .
\end{aligned}
$$

The penultimate convergent is

$$
m t+\frac{1}{2 t}=\frac{2 m t^{2}+1}{2 t} .
$$

Thus, we get the relation

$$
\left(2 m t^{2}+1\right)^{2}-\left(m^{2} t^{2}-m\right)(2 t)^{2}=1 .
$$

Similarly, (2)-(4) and (7) can all be proven.

Theorem 5. Suppose $D=16 k^{2} t^{2}+8 k^{2} t+k^{2}+k$, where $t, k$ are any natural numbers. Then:

(1) $a+b \sqrt{D}$ is the fundamental solution of (2) where $a=2 k(4 t+1)^{2}+1, b=2(4 t+1)$.

(2) If $k=1$, then $L+M \sqrt{D}$ is the fundamental solution of (3) where $L=4 t+1, M=1$.

Proof. (1) Expanding $\sqrt{D}$ into a continued fraction expansion, we obtain

$$
\sqrt{D}=[4 k t+k ; \overline{2(4 t+k), 2(4 k t+k)}] .
$$

The penultimate convergent is

$$
4 k t+k+\frac{1}{2(4 t+k)}=\frac{2 k(4 t+1)^{2}+1}{2(4 t+1)} .
$$

Thus, we get the relation

$$
\left(2 k(4 t+1)^{2}+1\right)^{2}-\left(16 k^{2} t^{2}+8 k^{2} t+k^{2}+k\right)(2(4 t+1))=1 .
$$

(2) For $k=1, D=16 t^{2}+8 t+2$. Now, expanding $\sqrt{D}$ into a continued fraction expansion, we obtain

$$
\sqrt{D}=[4 t+1 ; \overline{2(4 t+1)}]
$$

By Proposition $1, \quad L=4 t+1, M=1$; then, $(4 t+1)+\sqrt{16 t^{2}+8 t+2}$ is the fundamental solution of (3).

Theorem 6. Suppose $D=t^{2}+2 c t+c^{2}+1$, where $t$, c are any natural numbers. Then:

(1) $L+M \sqrt{D}$ is the fundamental solution of (3) where $L=t+c, M=1$.

(2) $a+b \sqrt{D}$ is the fundamental solution of (2) where $a=2(t+c)^{2}+1, b=2(t+c)$.

Example 1. Let $D=4 t^{2}+2$; then, $\left(4 t^{2}+1\right)+2 t \sqrt{4 t^{2}+2}$ is the fundamental solution of (2), by (1) in Theorem 3 .

Example 2. Let $D=t^{2}+4 t+5$; then, $(t+2)+\sqrt{t^{2}+4 t+5}$ is the fundamental solution of (3) and $\left(2(t+2)^{2}+1\right)+2(t+2) \sqrt{t^{2}+4 t+5}$ is the fundamental solution of (2), by Theorem 5 .

\section{Data Availability}

No data were used to support this study.

\section{Conflicts of Interest}

The authors declare that they have no conflicts of interest.

\section{Acknowledgments}

We would like to thank Ahmad Issa for helpful discussions.

\section{References}

[1] M. B. Nathanson, "Polynomial pell's equations," Proceedings of the American Mathematical Society, vol. 56, no. 1, p. 89, 1976.

[2] R. A. Mollin, K. Cheng, and B. Goddard, "Pellian polynomials and Perion lengths of continued fractions," Serdica Mathematical Journal, vol. 27, pp. 317-342, 2001. 
[3] T. Nagell, Introduction to Number Theory, Wiley, Hoboken, NY, USA, 1951.

[4] J. Mclaughlin, "Polynomial solutions to Pell's equation and fundamental units in real quadratic fields," Journal of the London Mathematical Society, vol. 67, no. 1, pp. 16-28, 2018.

[5] A. M. S. Ramasamy, "Polynomial solutions for the Pell's equation," Indian Journal of Pure and Applied Mathematics, vol. 25, pp. 577-581, 1994.

[6] D. E. Flath, Introduction to Number Theory, Wiley, Hoboken, NY, USA, 1989. 